\title{
Capacity Analysis of Route Sink Networks Based on Time-sharing Transmission Protocol
}

\author{
LIN Nan \\ College of Software Technology, Zheng Zhou University, Henan Zhengzhou, 450002, China
}

Keywords: Wireless Ad-Hoc Network, Route Sink Networks, Energy Constraint, Time-sharing Transmission

\begin{abstract}
A capacity analysis of route sink networks based on time-sharing transmission protocol is proposed to solve the problem. Firstly, the effects of energy constraints on the capacity of wireless ad hoc networks are studied. The network has route sink function, and the node transmission process is based on time-sharing transmission protocol. It is assumed that node transmission is triggered when the energy is big enough in cache capacity and a capacity analysis model based on the closed queue network is proposed. The proposed model considers the details of data, energy buffers and random access protocol, simultaneously. Then the impacts of energy constraints on the random access protocol parameters are analyzed so as to optimize the network performance.
\end{abstract}

\section{Introduction}

In order to decrease energy consumption in line with green objectives, it is necessary to plan and deploy the base station infrastructure. According to green communications, researchers focus on how to utilize the energy to realize the communication effectively[1]. Specifically, the so-called green communications is desired to adopt clean energy instead of fossil chemical energy mechanisms [2] [3]. Therefore, energy problem is an important factor in a wireless communication system[4]. For example, considering the ad-hoc networks, the environmental parameters need to configure a solar cell or energy search circuit so as to detect the source of energy in the natural environment [5] [6] [7] [8]. Due to the limited battery energy for sensor, a sink node is needed to replenish energy for transmitting and exchanging data[9] [10].

It is considered that wireless ad hoc networks have a finite number of nodes, and each node is within the coverage of other nodes. Medium access control protocol adopts time-sharing protocol, so that each wireless node will wait a random amount of slots before transmitting data packet. Therefore, the inhibitor of the increased throughput is dominated by the probability of collision. Under this network environment, asymmetrical nodes indicate that the arrival rate and the size of the contention window may be different. Each node has a energy buffer with limited capacity. Based on a closed queuing network theory, a new analytical model is established to assess the impact of the window size and the energy reachable rate on different networks.

\section{Analytical model based on queuing theory}

It is considered that the routing sink nodes can be modeled as a closed queuing network in the network topology,. The node in queuing network indicates the transmission status of head packet in data cache and the number of energy unit in energy cache, where transmission status includes idle state (without packets in data cache ), random waiting (with MAC mechanism), waiting packet with the limited energy and communication links. In addition, only one customer in queuing network can perform the performance change between different states in different transition probability, which is regarded as a Markov process. Each node is marked as $(j, X)$, where $j(j=0,1, \ldots, N)$ denotes the energy unit in energy cache, $X$ denotes the type of queuing node; it can be written as $I, T$ and $W_{k}(k=1,2, \ldots, m)$, which respectively denote idle (data buffer is empty), transmission (route sink node transmits data packet) and the first $k$ waiting time-slots (the first $k$ waiting time-slot before packet 
transmission). Node $E W$ denotes the waiting stage of reachable energy. In the next section, we will introduce how the customer realizes the transitions between the states, and compute the transition probability and service time.

Taking into account of the time-sharing characteristic of MAC protocol, the packet transmission and the waiting process are initializingd to MRF process in the time slot. The service time of idle state is a time slot. Thus, if no packet in last time-slot arrives on the sink node at the end of the service time, customers will leave in idle state $(j, I)$ and move to the idle state in a queuing network. If non-energy is reached, the customer moves to the previous idle state, otherwise the object idle state corresponds to the first waiting time slot $\left(W_{1}\right)$ according to the number of energy reachable unit. Therefore, service time and transition probability of idle state $(j, I)$ can be denoted as follows:

$$
\begin{gathered}
\operatorname{Pr}_{(j, I) \rightarrow(l, I)}^{i}=p_{0}\left(\sigma \lambda^{i}\right) p_{l-j}\left(\sigma \gamma^{i}\right) \\
l=j, \ldots, N-1, j=0, \ldots, N-1 \\
\operatorname{Pr}_{(j, I) \rightarrow(N, I)}^{i}=p_{0}\left(\sigma \lambda^{i}\right)\left(1-\sum_{l=0}^{N-j-1} p_{l}\left(\gamma^{i} \sigma\right)\right) \\
j=0, \ldots, N \\
T_{(j, I)}^{i}=\sigma, j=0, \ldots, N
\end{gathered}
$$

where, $p_{k}(x)=\frac{x^{k} e^{-x}}{k !}, \sigma$ is the slot length, $\gamma^{i}$ and $\lambda^{i}$ are reachable rate of data packet and energy units, respectively. In addition, $\operatorname{Pr}_{Y \rightarrow Z}^{i}$ and $T_{Y}^{i}$ respectively denotes transition probability from state $\mathrm{Y}$ to state $\mathrm{Z}$ and status service time of state $\mathrm{Y}$. The first factor on the middle right side indicates there is no reachable rate of data packet within a time slot. And the second factor for reaching $l-j$ energy units is the probability within a time slot. Therefore, customers are transferred from the state $(j, I)$ to the state $(l, I)$. Equation shows that if there are $N-j$ energy units arrive within a time slot, customers are transferred to the $(N, I)$ for the limited energy cache, The equation also indicates that if no data packet arrived, no matter how much energy unit number arriving, the customer will be transferred from the state $(N, I)$ to state $(N, I)$. In the total sum, the first multiplier factor denotes the reachable probability at least one data packet within the time slot. For each random wait state, namely state $\left(j, W_{k}\right)(j=0, \ldots, N, k=1, \ldots, m)$, the service time is equal to the length of a time slot. After the customer left a random wait state, if random wait time is not over, the customer will move to the next random wait state, from $\left(j, W_{k}\right)$ to $\left(l, W_{k+1}\right)$. Otherwise, it is transferred to the delivery status. Obviously, if the system has enough energy, it can be directly transferred to the delivery status. If the customer left a wait state, energy cache has non-energy unit (state $\left(0, W_{k}\right)$ ), while there is non-energy unit arriving in service time of this state, then the group is moved to the waiting state $E W$, until an energy unit arrives. Therefore, the transition probability from waiting states to the other state can be denoted as follows:

$$
\begin{aligned}
& \operatorname{Pr}_{\left(j, W_{k}\right) \rightarrow\left(l, w_{k+1}\right)}^{i}=\frac{m-k}{m-k+1} p_{l-j}\left(\gamma^{i} \sigma\right) \\
& l=j, \ldots, N-1, j=0, \ldots, N-1, k=1, \ldots, m-1
\end{aligned}
$$

In the last two types, the first multiplier factor is the probability happening after the finished $k$ waiting time slot, but the waiting time is still not over and continues for at least one time slot, which is the probability where customers are transferred from waiting state to state $E W$ and delivery status:

$$
\begin{aligned}
\operatorname{Pr}_{\left(j, W_{k}\right) \rightarrow(N, T)}^{i} & =\frac{1}{m-k+1}\left(1-\sum_{l=0}^{N-j-1} p_{l}\left(\gamma^{i} \sigma\right)\right) \\
j & =0, \ldots, N, \quad k=1, \ldots, m
\end{aligned}
$$

According to the above analysis, state $E W$ has the characterization of the waiting time due to a lack of energy, and its service time is length of time slot. After the customer leaves the state, if there is no energy storage, the customer will still return to the state $E W$. Otherwise, the customers in state $E W$ are transferred to the delivery status. Therefore, transition probability of customer in the state $E W$ is shown as follows: 


$$
\begin{gathered}
\operatorname{Pr}_{E W \rightarrow E W}^{i}=p_{0}\left(\gamma^{i} \sigma\right) \\
\operatorname{Pr}_{E W \rightarrow(l, T)}^{i}=p_{l}\left(\gamma^{i} \sigma\right), l=1, \ldots, N-1
\end{gathered}
$$

If customers are in the state $(j, T)(j=1, \ldots, N)$ at the beginning of time slot, there are $j$ unit energy storage, which needs one energy unit to send data. At the end of the sending, if the packet has collision, the customer will move to the first random waiting time slot to start the random waiting processes. Similarly, when the packet sent successfully and at least one data packet in data cache, the customers are transferred to the first random waiting time slot to start the new packet processing. If successful packet data cache is empty, the customers can be transferred into the idle state according to the energy unit quantity. Since the assumption data obeys the Poisson distribution, they are sent in sequence, and therefore routing convergence node can be regarded as a M/G / 1 queue. According to the queuing theory after a customer left delivery status, empty probability of the observed queue is $\rho^{i}$, where $\rho^{i}$ is the business strength for the node. Based on this introduce and analysis, the transition probability of customers from the sent state can be written as:

$$
\begin{gathered}
\operatorname{Pr}_{(j, T) \rightarrow\left(l, W_{1}\right)}^{i}=\left(P_{c o l}^{i}+\rho^{i}\left(1-P_{c o l}^{i}\right)\right) p_{l-j+1}\left(\sigma \gamma^{i}\right) \\
l=j-1, \ldots, N-1, j=1, \ldots, N \\
\operatorname{Pr}_{(j, T, I) \rightarrow(N, I)}^{i}=\left(1-\rho^{i}\right)\left(1-P_{c o l}^{i}\right)\left(1-\sum_{l=0}^{N-j} p_{l}\left(\sigma \gamma^{i}\right)\right) \\
j=1, \ldots, N
\end{gathered}
$$

where $P_{c o l}^{i}$ is the probability of collision when the packet is sent.

So apart from idle, other state sum of the probability in queuing network is the non-empty routing convergence probability, which is the business strength of routing convergence nodes. so the analytical equation can be written as follows,

$$
\rho^{i}=\sum_{l=0}^{N} \sum_{k=1}^{m} \rho_{\left(l, W_{k}\right)}^{i}+\sum_{l=1}^{N} \rho_{(l, T)}^{i}+\rho_{E W}^{i}+\rho_{I^{\prime}}^{i}
$$

when the packet arrived in the convergence nodes, if the nodes are idle, then this packet need to wait until the next time slot. The waiting time needs be included in the service time of packet. Otherwise there will be packets in the queue, but there is also idle in the queue. So the $\rho_{I^{\prime}}^{i}$ refers to the occupancy probability of routing convergence node caused by waiting for the next time slot. According to Poisson arrival process knowledge, the average residence time is $0.5 \sigma$.Similarly, all reaching free routing convergence node packets will lead to reside, so the arrival rate of queuing network idle state can be calculated for $\rho_{I^{\prime}}^{i}$. Therefore, $\rho_{I^{\prime}}^{i}$ can be written as $(0.5 \sigma) \sum_{l=1}^{N}\left(1-\rho^{i}\right)\left(1-P_{c o l}^{i}\right) a_{(l, T)}^{i}$, where $a_{(l, T)}^{i}$ means customer will have state $(l, T)$ probability of the customers transit from the state $(l, T)$ to an idle state in the queuing network written as $\left(1-\rho^{i}\right)\left(1-P_{\text {col }}^{i}\right)$.

The probability of customers staying on the delivery status, is actual proportion of sending time when routing convergence nodes are under steady state. Since the average length of service time for all states in the queuing network are time slots, the proportion is the sending probability after routing stabilization of convergence node is emphasized at the beginning of time slot. So the routing sending probability $P_{t x}^{i}$ of convergence nodes can be denoted as

The collision probability can be denoteddenoted as follows

$$
P_{t x}^{i}=\sum_{l=1}^{N} \rho_{(l, T)}^{i}
$$

$$
P_{c o l}^{i}=1-\prod_{l=1, l \neq i}^{n}\left(1-P_{t x}^{i}\right)
$$

In order to maintain all nodes stability, maximum stable throughput can be denoted as maximum arrival rate of routing convergence nodes, which is denoted as $\Lambda_{\text {st }}$ and is written as follows,

$$
\Lambda_{s t}=\max \lambda \text { s.t. } \forall i: 1 \leq i \leq n, \rho^{i}<1
$$




\section{Simulation results and analysis}

According to the simulation experiment, this section is to analyze the energy constraints impact on network performance indicators. At the same time, the results of our proposed algorithm will be compared with monte carlo simulation results, to illustrate the validity of the proposed model in this paper. The time slot length for assumption simulation is $\sigma=1 \mathrm{~s}$, and its energy cache size is 50 .

Figure 1 shows the maximum stable throughput in different energy reaching rate under the two competition windows. Given a fixed competition windows, as energy arrival rate increases, the largest stable throughput will increase, due to more energy units to support packet sending.

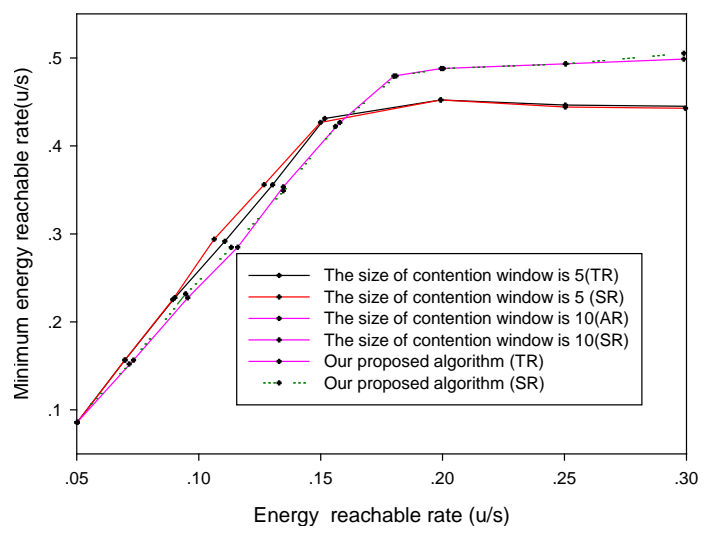

Figure 1. Energy reachable rate

Figure 1 shows the relationship between the maximum stable throughput and energy rate. However, excessive packet sending rate will make collisions increasing, thus increasing energy rate cannot reach the increasing of the maximum stable throughput infinitely. Under the low energy arrival rate, packet is more likely to wait for the lack of energy. The maximum stable throughput is not changed by competition window size at this time. Under high-energy arrival rate, on the other hand, most of the packet can get energy unit, packet delay is for a collision, rather than for a lack of energy. Due to the competition window, the average service time is greater, and then the maximum stable throughput also is dropped. At the same time, the result of simulation shows that this paper analysis results has the accuracy and validity of our proposed algorithm.

Considering a network composed of two routing convergence nodes under different energy reachable rate, the stable domain changes along with the different packet arrival rate, as shown in figure 2 . If the packet reachable rate of one routing convergence node increases, then the biggest reachable rate of another node is also reduced to keep the stability of the network. This is because under the high data rate, the more collision results in service time and business strength increasing. In addition, pure time sharing protocol has more stable domain because there is non-energy constraints.

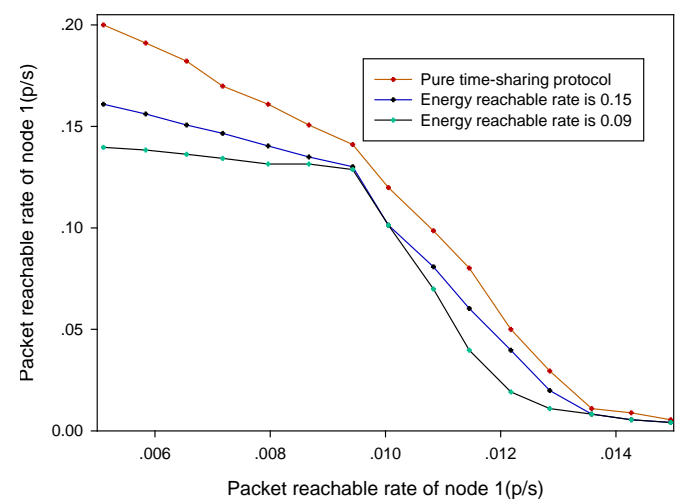

Figure 2. Stability packet reachable rate for two routing convergence nodes

Figure 3 shows the minimum energy arrival rate required to keep the low packet discard rate. The gathering nodes number is 5 , the size of competition window is 10 . In order to make the packet 
discard rate remains the same when packet arrival rate increases, the required minimum energy reachable rate will increase. Collision packets, in fact, in addition to external arrived ones, will be discarded too. This makes the energy required to keep the same packet discard rate increases.

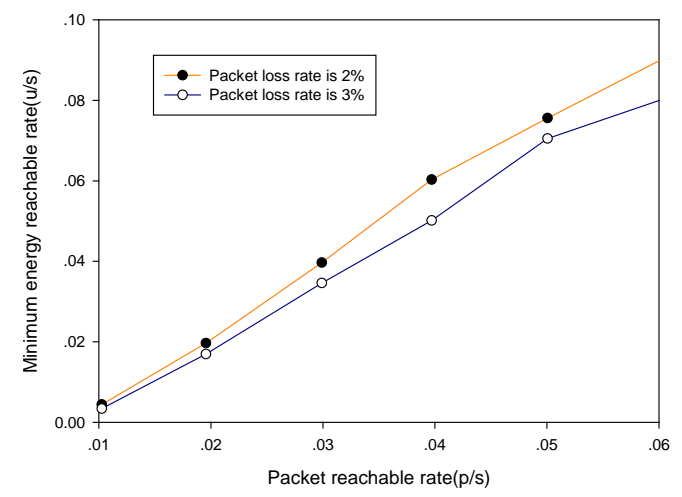

Figure 3. Minimum energy reachable rate

\section{Conclusion}

The node energy constraint in wireless ad-hoc network is the mainspring that researchers may largely adopt green energy. This paper proposes an analysis model applied to the analysis network throughput. Network connection based on time-sharing protocol has many routing convergence nodes. In this paper, our proposed models can be adopted as modeling for the different data packets, different energy arrival rate and the different quantity of unsaturated node scenarios. This article proposes the routing convergence nodes of network as closure queuing network, which is characterized by data sending processes and energy saving states network performance indicators injects the largest stable throughput, stable domain and available models with packet discard rate to analyze. Simulation experiments demonstrate how the energy constraints affect these performance indicators and validate the accuracy and validity of the proposed analysis model.

\section{References}

[1] Gao L, Zhang R, Yin C, et al. Throughput and delay scaling in supportive two-tier networks[J]. Selected Areas in Communications, IEEE Journal on, 2012, 30(2): 415-424.

[2]Andreou P, Zeinalipour-Yazti D, Pamboris A, et al. Optimized query routing trees for wireless sensor networks[J]. Information Systems, 2011, 36(2): 267-291.

[3]Climent S, Sanchez A, Capella J V, et al. Underwater Acoustic Wireless Sensor Networks: Advances and Future Trends in Physical, MAC and Routing Layers[J]. Sensors, 2014, 14(1): 795-833.

[4]Zhu G, Davis L M, Chan T. Energy-Throughput Trade-offs in a Wireless Sensor Network with Mobile Relay[J]. arXiv preprint arXiv:1403.5824, 2014.

[5]Ryu J, Ying L, Shakkottai S. Timescale decoupled routing and rate control in intermittently connected networks[J]. IEEE/ACM Transactions on Networking (TON), 2012, 20(4): 1138-1151.

[6]Khojasteh H, Mišić J, Mišić V B. Integration of an IEEE 802.15. 4 RFID network with mobile readers with a 802.11 WLAN[J]. Wireless Communications and Mobile Computing, 2012.

[7]Jabbar S, Ahmad A, Ikram A A, et al. TSEEC-TS/TDMA based Energy efficient Congestion Control in Mobile Wireless Sensor Network[C]//Proceedings of the World Congress on Engineering and Computer Science. 2011, 2: 19-21.

[8]Rubin I, Lin Y Y, Kofman D. Relay-aided networking for power line communications[C]//Information Theory and Applications Workshop (ITA), 2014. IEEE, 2014: 1-6. 
Attar H, Stankovic L, Stankovic V. Cooperative network-coding system for wireless sensor networks[J]. IET communications, 2012, 6(3): 344-352.

[9]Mitchell P D, Qiu J, Li H, et al. Use of aerial platforms for energy efficient medium access control in wireless sensor networks[J]. Computer Communications, 2010, 33(4): 500-512.

[10]Pandey R, Ghosh R. Apparatus and method for managing packet routing through internally-powered network devices in wireless sensor networks: U.S. Patent 8,532,003[P]. 2013-9-10. 